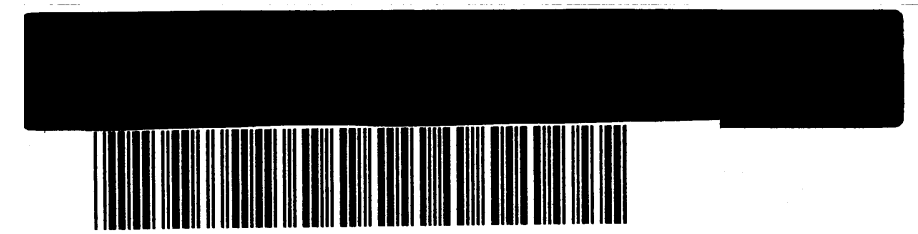

CERN-EP/90-88

20 June 1990

\title{
CM-P00056461
}

\section{REFLECTIVE UV PHOTOCATHODES WTTH GAS-PHASE ELECTRON EXTRACTION: SOLID, LIQUID, AND ADSORBED THIN FILMS}

\author{
J. Séguinot 1), G. Charpak ${ }^{2)}$, Y. Giomataris ${ }^{2)}$, V. Peskov ${ }^{3)}$, J. Tischhauser ${ }^{2)}$ and T. Ypsilantis ${ }^{1)}$
}

1) Collège de France, Paris, France.

2) CERN, Geneva, Switzerland.

3) World Lab., Geneva, Switzerland.

\section{ABSTRACT}

The photoemission quantum efficiency of reflective photocathodes in methane gas has been investigated in the spectral range between 140 and $250 \mathrm{~nm}$. The spectral response of solid metal and CsI, as well as of liquid and solid TMAE film, has been measured. The high quantum efficiency of CsI $(35 \%$ at $170 \mathrm{~nm})$ makes it attractive for $\mathrm{BaF}_{2}$ or xenon scintillation detection. $\mathrm{A} \mathrm{BaF}_{2}$ crystal coupled to an ionization chamber with a reflective CsI photocathode has been successfully tested. Adsorbed TMAE films can significantly increase the quantum yields of metal and CsI (to $46 \%$ at $170 \mathrm{~nm}$ ), making them suitable for fast RICH and other applications.

(Submitted to Nuclear Instruments and Methods

in Physics Research A) 


\section{INTRODUCTION}

Investigations of organic reflective UV photocathodes with electron extraction into a gaseous medium were reported in an earlier article [1]. The molecules studied were those of liquid tetrakis(dimethylamine)ethylene (TMAE) and of tetramethyl-p-phenylenediamine (TMPD). Most of these measurements were carried out in the spectral region of wavelength $\lambda>230 \mathrm{~nm}$ so as to exclude the masking effects of gas-phase photoionization. The quantum efficiency was always found to increase with photon energy, and extrapolation to $\lambda \cong 180 \mathrm{~nm}$ indicated that large $(\sim 10 \%)$ efficiencies were possible (see figs. $8 \mathrm{~b}$ and 11 in ref. [1]).

In order to verify this extrapolation, we have investigated some reflective solid/liquid/adsorbed-film photocathodes in the region between 140 and $250 \mathrm{~nm}$. An apparatus was designed to deposit photocathodes by cryopumping a gas onto a cold metal substrate. After deposition, the flow of the photosensitive gas could be stopped, thus permitting photoefficiency measurements to be made with minimum interference from gas-phase photoionization. However, there always remained the effect of the equilibrium vapour pressure of the photosensitive molecule, which could be removed-in order to isolate the intrinsic photocathode effect-by making an appropriate set of measurements. The data show that the quantum yields are indeed high. One particular photocathode (solid CsI + adsorbed TMAE film) has $46 \%$ efficiency at $170 \mathrm{~nm}$. Such a cathode can have important applications in the field of high-energy physics, i.e. for fast RingImaging CHerenkov (RICH) detectors [2], for $\mathrm{BaF}_{2}$ calorimetry [3], and for liquid-xenon electromagnetic calorimetry [4]. In addition, it may find uses in Positron Emission Tomography (PET), either with $\mathrm{BaF}_{2}$ or liquid xenon.

A preliminary report of these results was presented in September 1989 at the ECFA Conference held in Barcelona and was published in the Proceedings [5].

\section{EXPERIMENTAL SET-UP}

The apparatus is shown in fig. 1a. It consists of a deuterium-filled low-pressure d.c. arc lamp with a $\mathrm{MgF}_{2}$ window (labelled La in the figure; Hamamatsu L879-01); a UV photon monochromator (PMC; Jobin-Yvon H20VUV), and a photoefficiency test chamber (TC). The wavelength-selected photon beam is parallel-focused by an $\mathrm{f}=100 \mathrm{~mm}, \phi=25 \mathrm{~mm} \mathrm{CaF}_{2}$ lens (Le) placed $100 \mathrm{~mm}$ downstream from the monochromator exit slit. This parallel beam is then split into a reflected beam and a transmitted beam by means of a flat, $5 \mathrm{~mm}$ thick, $\mathrm{CaF}_{2}$ crystal beam-splitter (BS) tilted $45^{\circ}$ to the beam direction. The reflected beam is monitored by a photomultiplier (PMR; RCA 8850), whose glass entrance window is coated (by vacuum deposition) with a p-terphenyl layer of wavelength shifter, $10^{4} \AA$ thick [6], protected by a $10^{3} \AA$ thick layer of $\mathrm{MgF}_{2}$. An identical photomultiplier (PMT; RCA 8850) is positioned in such a way as to measure the transmitted photon beam (see fig. 1a). The $5 \mathrm{~mm}$ diameter beam enters the test chamber through a flat, $5 \mathrm{~mm}$ thick, $\mathrm{CaF}_{2}$ window $(\mathrm{W})$, which isolates the monochromator from the TC. This guarantees that the light source is unaffected by any contaminants originating in the TC. 
The test chamber is shown in more detail in fig. 1b. It consists of a horizontal (z-direction) stainless-steel cylinder of $100 \mathrm{~mm}$ diameter with a transverse vertical (y-direction) cylinder positioned centrally to form a cross. The horizontal cylinder is linked by one end-flange to the photon monochromator (PMC), whilst the other end-flange is linked to the photomultiplier (PMT). The test chamber contains an ionization cell composed of a transparent mesh cathode (MC) plane, an anode (A) wire plane, followed by the solid photocathode (PC) plane and a cold trap. The cold trap can be cooled to a temperature much lower than that of the photocathode, so as to trap most of the impurities coming from the TC ambient-temperature walls. This facility was used to test operation of CsI photocathodes down to $160 \mathrm{~K}$.

The PC plane is made of a stainless-steel (ss) plate ( $\Delta y=45 \mathrm{~mm}$ high, $\Delta x=22 \mathrm{~mm}$ across, and $\Delta z=0.5 \mathrm{~mm}$ thick) with a $4 \mathrm{~mm}$ diameter ss tube soldered around its perimeter. The ss plate can be cooled to $\geq 120 \mathrm{~K}$ by flowing cold $\mathrm{N}_{2}$ gas through the tube by means of an evacuated transfer line. Separate plates of brass, copper, or stainless steel can be clipped onto the PC plane (with good thermal contact), thus permitting us to study these bare metals easily. These plates can also be transported to a separate vacuum deposition facility for coating with various low-vapour-pressure materials (CsI, $\mathrm{Zn}$, etc.) for subsequent measurement in the TC. The temperature of the PC plane is continuously monitored by means of a calibrated platinum resistor. The PC plane itself can be raised vertically by $\Delta y=50 \mathrm{~mm}$ (without affecting the gas flow or the temperature), allowing the photon beam to impinge directly onto the monitor PMT. The PC potential $\mathrm{V}_{\mathrm{PC}}$ is negative and variable between $0 \leq-\mathrm{V}_{\mathrm{PC}} \leq 3 \mathrm{kV}$.

The anode or collector plane (A) consists of three $2 \mathrm{~mm}$ diameter vertical wires spaced $10 \mathrm{~mm}$ apart in the transverse horizontal $(x)$ direction. The middle wire $(x=0)$ is kept at ground potential by a digital electrometer (Keithly 616A; lower current limit $=0.01 \mathrm{pA}$ ). It collects all electrons coming from the target region of the PC $(-5 \leq x \leq 5 \mathrm{~mm})$, whilst the two edge wires $(x=+10 \mathrm{~mm}$ and $x=-10 \mathrm{~mm}$ ) are independently grounded so as to define completely the electrostatic configuration of the ionization cell.

The mesh cathode $(\mathrm{MC})$ potential $\mathrm{V}_{\mathrm{MC}}$ is also negative and variable between 0 and $2 \mathrm{kV}$. It serves to define the fiducial collection region for gas-phase photoionization, as well as to ensure that all lines of force from the cathode terminate on the collection anode.

Before assembly, all the metallic elements of the TC were cleansed by sequential washing in acetone, detergent, and distilled water, then pumped and outgassed at $200{ }^{\circ} \mathrm{C}$.

The PMC is continuously flushed with argon (quality N48; $20 \mathrm{ppm}$ impurities) at atmospheric pressure. The window (W) isolates the PMC from the variable conditions in the TC. The test chamber itself is flushed independently, usually with methane (quality N35; 500 ppm impurities), or sometimes with argon (N48). The flushing gases are cleansed by passing them through a cartridge of Oxisorb (chrome trioxide, silica gel, and molecular sieves; Messer Griesham $\mathrm{GmbH}$ ) before entering either the PMC or the TC. The TC flushing gas can be saturated at a partial vapour pressure of an organic photosensitive molecule by bubbling a fraction of the carrier gas 
through a vessel containing the aforesaid molecules in the liquid state and keeping it at $18{ }^{\circ} \mathrm{C}$ in order to adjust the photon mean free path to about half the distance between the entrance window and the PMT. In order to prevent any risk of condensation of the photosensitive molecules onto the PMT wavelength shifter, the PMT was heated to about $50^{\circ} \mathrm{C}$. The TC exhaust is discharged into air through a stainless-steel capillary ( $3 \mathrm{~m}$ long, $2 \mathrm{~mm}$ in diameter) so as to limit the retrodiffusion of oxygen.

The distances between the entrance window (W) and the mesh cathode $(\mathrm{MC})$, the collection anode (A), the photocathode (PC), and the phototube (PMT) are $\mathrm{z}_{\mathrm{MC}}=116, \mathrm{z}_{\mathrm{A}}=129, \mathrm{z}_{\mathrm{PC}}=141, \mathrm{z}_{\mathrm{T}}=$ $236 \mathrm{~mm}$ respectively.

\section{MEASUREMENTS}

\subsection{Flux calibration}

A current measurement is used to determine the photon flux, because of its greater linear dynamic range compared with photon counting.

Initially, the TC is flushed with methane at atmospheric pressure, and the measurements begin only when optimal photon transmission is obtained (at $\lambda=160 \mathrm{~nm}$ ). The measurement cycle starts with a calibration of the transmitted photon beam relative to the reflected photon beam. The reflected and transmitted photocurrents $I_{R}$ and $I_{T}(0)$ are measured (between 140 and $250 \mathrm{~nm}$ ) with $1 \mathrm{~mm}$ monochromator slits $\left(\sigma_{\lambda}=4.3 \mathrm{~nm}\right)$ in order to maximize the sensitivity. This increased sensitivity is especially necessary at the longest wavelengths, where the yield is small. At each wavelength $\lambda$, the photocurrents are determined by the following relations

$$
\begin{array}{ll}
I_{R} & =\mathrm{eI}_{0} R_{B S} Q_{R} G_{R^{\prime}} \\
I_{T}(0) & =\mathrm{eI}_{0}\left(1-R_{B S}\right) Q_{T} G_{T}, \\
C \quad & =I_{T}(0) / I_{R},
\end{array}
$$

where $e$ is the electron charge; $I_{0}$ is the incident photon flux, $Q_{T}, Q_{R}$, and $G_{T}, G_{R}$ are the transmittive and reflective photomultiplier quantum efficiencies and gains, respectively; and $R_{B S}$ is the reflectivity of the beam splitter. The argument $(0)$ in the above equations means that there is no photoionizing gas in the test chamber. The wavelength dependence is explicit. The quantity $C$ calibrates the transmitted beam current $I_{T}(0)$ in terms of the reflected beam current $I_{R}$. This current is measured before the beam enters the TC; hence it is independent of the gas conditions in the TC (i.e. without the argument).

When a photoionizing (PI) gas is introduced into the TC, a photoionization current $I_{A}(P I)$ is observed on the anode wire. It is given by the relation

$$
I_{A}(P I)=\mathrm{eI}_{0} Q_{P I} A_{P I}\left(z_{M C}, z_{P C}\right),
$$

where 


$$
A_{P 1}\left(z_{M C} z_{P C}\right)=\exp \left(-z_{M C} / 1_{p h}\right)-\exp \left(-z_{P C} / 1_{p h}\right)
$$

and $Q_{P I}$ is the gas-phase photoionization quantum efficiency. The quantity $A_{P I}\left(z_{M C}, z_{P C}\right)$ is the absorptivity of the PI gas in the ionization cell between $\mathrm{z}_{\mathrm{MC}}$ and $\mathrm{z}_{\mathrm{PC}}$. The photon absorption length $\left(l_{p h}\right)$ is determined by measuring the current $I_{T}(P I)$, which reaches the PMT when the photoionizing gas is in the TC and (necessarily) with the PC in the 'up' position. The equation for this current is then determined by the TC absorptivity as

$$
I_{T}(P I)=e I_{0} Q_{T} G_{T} \exp \left(-z_{T} / 1_{p h}\right)=I_{T}(0) \exp \left(-z_{T} / l_{p h}\right)
$$

whilst from eq. (1), $I_{T}(0)=C I_{R}$; hence

$$
\mathrm{I}_{\mathrm{ph}}=\mathrm{z}_{\mathrm{T}} / \ln \left[\mathrm{C} \mathrm{I}_{\mathrm{R}} / \mathrm{I}_{\mathrm{T}}(\mathrm{PI})\right]
$$

The incident photon flux $\mathrm{I}_{0}$ may now be calculated from eq. (2) as

$$
I_{0}=I_{A}(P I) /\left[e Q_{P 1} A_{P I}\left(z_{M C}, z_{P C}\right)\right]
$$

where $I_{A}(P I)$ is measured directly, $Q_{P I}$ is from the known PI efficiency, i.e. for TMAE $[7,8] A_{P I}$ is from eq. (3), and $\mathrm{l}_{\mathrm{ph}}$ is from eq. (5).

Having evaluated $l_{p h}$ and $I_{0}$, the product $Q_{T} G_{T}$ may then be evaluated from eq. (4). The gain $G_{T}$, independent of the wavelength, is given by the manufacturer as $g=1.4 \times 10^{5}$ at the chosen operating voltage of $1.4 \mathrm{kV}$. The quantity $\mathrm{Q}_{\mathrm{T}} \mathrm{G}_{\mathrm{T}} / \mathrm{g}$, plotted in fig. $2 \mathrm{a}$, gives the quantum efficiency of the PMT if the gain $g$ is, indeed, correct. The average PM quantum efficiency is $\sim 10 \%$, not inconsistent with previous measurements [6], but with a surprising (i.e. not flat) wavelength dependence, which is probably due to a variation in the p-terphenyl wavelength-shifter efficiency. It must be emphasized, however, that the derived PC quantum yield depends only on the TMAE photoionization yield and not on the PMT quantum efficiency, it being used only as an intermediate standard. A measurement of the product $Q_{T} G_{T}$ was still performed above $200 \mathrm{~nm}$, by using a calibrated photodiode EGG UV $360 \mathrm{BQ}$. A good agreement was obtained in the region of overlap with the TMAE, between 200 and $220 \mathrm{~nm}$, confirming the validity of the method.

\subsection{Photocathode efficiency}

When measuring a photocathode that has a negligible equilibrium vapour pressure, the current $I_{A}(P C)$ on the wire anode $(A)$ is given by the obvious relation

$$
\mathrm{I}_{\mathrm{A}}(\mathrm{PC})=\mathrm{eI}_{0} \mathrm{Q}_{\mathrm{PC}}
$$


Solving for the PC quantum efficiency, with $\mathrm{I}_{0}$ from eq. (6), gives

$$
Q_{P C}=Q_{P I} A_{P I}\left(z_{M C}, z_{P C}\right)\left[I_{A}(P C) / I_{A}(P I)\right],
$$

where $A_{P I}$ is from eq. (3). The PC quantum efficiency thus depends on the PI gas quantum efficiency, on the measured currents, and on the absorptivity (i.e. $l_{\mathrm{ph}}$ ), and is independent of the PM quantum efficiency and gain.

When measuring a photocathode that has non-negligible vapour pressure, the anode signal $I_{A}$ will have a component arising from gas-phase photoionization as well as from the PC. In this case, the procedure is to measure the anode current $I_{A d}$ with the $P C$ in the down position (so as to intercept the beam), and the current $I_{A u}$ when the $P C$ is in the up position. In this latter position a current $\mathrm{I}_{\mathrm{T}}$ may also be observed in the PMT, which is due to unabsorbed photons reaching the PMT. Note that when the $\mathrm{PC}$ is in the up position the ionization cell becomes much longer because the PMT cathode is also at a negative potential $(-1.4 \mathrm{kV})$, and charge collection occurs between $\mathrm{z}_{\mathrm{MC}}$ and $\mathrm{z}_{\mathrm{T}}$. It has been verified that the photoionization charge is efficiently collected in this region at the applied field of $150 \mathrm{~V} / \mathrm{cm}$ (i.e. $1.4 \mathrm{kV}$ over $9.5 \mathrm{~cm}$ ), provided $\left|\mathrm{V}_{\mathrm{PC}}\right|$ is higher than $1.0 \mathrm{kV}$, as shown in fig. $2 b$. This current determines the photon absorption length $l_{p h}$, which then allows the photoeffects due to the PC and those due to the PI gas to be separated. The observed currents are

$$
\begin{aligned}
I_{A d}=I_{A}(P C)+I_{A}(P I) & =I_{0}\left[Q_{P C} \exp \left(-z_{P C} / l_{p h}\right)+Q_{P I} A_{P I}\left(z_{M C} z_{P C}\right)\right] \\
I_{A u} & =\mathrm{eI}_{0} Q_{P I} A_{P I}\left(z_{M C}, z_{T}\right) \\
I_{T}(P I) & =e_{0} \exp \left(-z_{T} / I_{P h} Q_{T} G_{T} .\right.
\end{aligned}
$$

Combining the first two relations of eq. (9) gives the key equation for the PC quantum efficiency:

$$
Q_{P C}=Q_{P I}\left(A d / I_{A u}\right)\left[\exp \left(z_{1} / 1_{p h}\right) \exp \left(-z_{2} / 1_{p h}\right)\right]+1-\exp \left(z_{1} / 1_{p h}\right)
$$

where $z_{1}=z_{P C}-z_{M C}$ and $z_{2}=z_{T}-z_{P C}$. The third relation of eq. (9), with $I_{T}(0)$ from eq. (1), determines $1_{\mathrm{ph}}$ exactly as in eq. (5). Here, as before, the PC quantum efficiency depends on the PI gas quantum efficiency, on the measured currents, and on $\mathrm{l}_{\mathrm{ph}}$, and is independent of the PMT quantum efficiency or gain.

The final results are independent of any intensity variations in the lamp or in the monochromator throughput because all measurements are normalized to the reflected photon current $\mathbf{I}_{\mathbf{R}}$. 


\section{EXPERIMENTAL RESULTS}

\subsection{Metal photocathodes}

The quantum efficiencies of bare ss, copper, and brass PC plates have been measured, and the results are shown in fig. 3. The quantum yields relative to TMAE are determined from eq. (8). As expected, the yields are low $\left(\leq 10^{-4}\right)$ for $\lambda \geq 185 \mathrm{~nm}$ and in the ss case reach only $0.4 \%$ at $\lambda=$ $145 \mathrm{~nm}$.

It was expected that, of the non-reactive metals, a zinc (or cadmium) photocathode would have the highest yield, reaching $0.7 \%$ at $200 \mathrm{~nm}$ [9]. For this reason, a $2 \mu \mathrm{m}$ layer of zinc (Ventron; impurities $\leq 10 \mathrm{ppm}$ ) was vacuum-deposited on a PC plate. The measured yield, shown in fig. 3 , is only a factor of 2 larger than that of the copper cathode and much less than the value given in ref. [9]. However, the zinc surface was exposed to air for about $10 \mathrm{~min}$, hence some oxidation might have occurred.

\subsection{Solid-TMAE photocathodes}

A solid-TMAE photocathode was prepared by flowing clean methane gas through a TMAE bubbler at a temperature of $293 \mathrm{~K}$, and then into the test chamber with the PC plate cooled to $260 \mathrm{~K}$. The TC walls and the PMT were maintained at $298 \mathrm{~K}$. The melting point of TMAE is $269 \mathrm{~K}$, its boiling point is $453 \mathrm{~K}$, and its equilibrium vapour pressure is

$$
p=p_{0} \exp \left[(\Delta H / R)\left(T_{0}^{-1}-T^{-1}\right)\right],
$$

where the factor $\Delta H / R=6370 \mathrm{~K}$, and $p_{0}=0.55$ Torr is the reference pressure at the reference temperature $T_{0}=300 \mathrm{~K}[10,11]$. A reduced TMAE concentration is obtained, when necessary, by mixing the TMAE with pure methane in adjustable proportions via calibrated mass flowmeters.

The $160 \mathrm{~nm}$ light transmission was monitored by the PMT as a function of time after TMAE injection, as shown in fig. 4. The transmission (TR) decreased for $8 \mathrm{~min}$, as the PI gas filled the TC, then became constant for $12 \mathrm{~min}$, after which time the TMAE injection was stopped. The constant value $\mathrm{TR}_{\mathrm{PC}}$ determines the equilibrium photon mean free path $\mathrm{l}_{\mathrm{PC}}$ of the cryopumped TMAE gas, i.e. $\mathrm{l}_{\mathrm{PC}}=-\mathrm{z}_{\mathrm{T}} / \ln \left(\mathrm{TR}_{\mathrm{PC}}\right)$. The corresponding equilibrium vapour pressure of TMAE is

$$
\mathrm{p}_{\mathrm{PC}}=\mathbf{k} \mathrm{T}_{\mathrm{PC}} / \sigma \mathbf{l}_{\mathrm{PC}}
$$

where $k$ is Boltzmann's constant $(=1.036 \mathrm{~mm} \cdot \mathrm{Torr} \cdot \mathrm{Mb} / \mathrm{K}), \mathrm{T}_{\mathrm{PC}}$ is the absolute temperature of the photocathode (and gas), and $\sigma$ is the photoabsorption cross-section $(=30 \mathrm{Mb})$ of TMAE at $\lambda=160 \mathrm{~nm}$.

The transmission of the same TMAE-laden gas in the TC and PC at ambient temperature $(298 \mathrm{~K})$ is also shown in fig. 4. In this case the transmission plateau is very small $\left(\sim 7.8 \times 10^{-4}\right)$, because the TMAE equilibrium vapour pressure is much higher without cryopumping. The mean free path $l_{\mathrm{ph}}$ at this temperature $T$, may be inferred from the slope of the curve, thus permitting the 
equilibrium vapour pressure of the non-cryopumped TMAE to be calculated analogously to eq. (12). The difference between these partial pressures determines the rate $(\mathrm{dn} / \mathrm{dt})$ at which TMAE molecules are cryopumped onto the cold PC, i.e.

$$
\Delta \mathrm{p}=\mathrm{p}-\mathrm{p}_{\mathrm{PC}}=(\mathrm{k} / \sigma)\left(\mathrm{T} / 1_{\mathrm{ph}}-\mathrm{T}_{\mathrm{PC}} / \mathrm{l}_{\mathrm{PC}}\right)
$$

and

$$
\mathrm{dn} / \mathrm{dt}=(\mathrm{dV} / \mathrm{dt}) \Delta \mathrm{p} / \mathrm{kT}_{\mathrm{PC}},
$$

hence

$$
\mathrm{dn} / \mathrm{dt}=(1 / \sigma)(\mathrm{dV} / \mathrm{dt})\left[\left(\mathrm{T} / \mathrm{T}_{\mathrm{PC}}\right) / 1_{\mathrm{ph}}-1 / 1_{\mathrm{PC}}\right]
$$

where $\mathrm{dV} / \mathrm{dt}$ is the volume flow-rate of the gas into the test chamber. Characteristic numbers are $\mathrm{dV} / \mathrm{dt}=10 \mathrm{l} / \mathrm{hr}, \mathrm{l}_{\mathrm{PC}}=10.7 \mathrm{~cm}$ at $\mathrm{T}_{\mathrm{PC}}=260 \mathrm{~K}, \mathrm{l}_{\mathrm{ph}}=3.3 \mathrm{~cm}$ at $\mathrm{T}=298 \mathrm{~K}$, and $\sigma=30 \mathrm{Mb}$, hence dn $/ \mathrm{dt}=$ $1.4 \times 10^{18}$ molecules per minute. The photocathode surface area is $2 \Delta x \times \Delta y=19.8 \mathrm{~cm}^{2}$, hence it has a molecular surface density of $7.1 \times 10^{16} \mathrm{molecules} / \mathrm{cm}^{2} \cdot \min$, which for $\sigma=30 \mathrm{Mb}$ corresponds to $2.11_{\text {ph }}$ deposited per minute. The TMAE molecular weight is 200 and its estimated solid density is $0.7 \mathrm{~g} / \mathrm{cm}^{3}$, giving a film deposit rate of $0.34 \mu \mathrm{m} / \mathrm{min}$. The deposit time was usually about $10 \mathrm{~min}$, giving a thickness $\left(\approx 3.4 \mu \mathrm{m}=211_{\mathrm{ph}}\right)$, which is more than sufficient to ensure that the photons are completely absorbed in the TMAE layer before they reach the metal substrate.

The collected anode current $I_{A}$ versus the PC potential $-V_{P C}$ is shown in fig. 5 for a solid photocathode with electron collection in pure methane. The current rises rapidly and becomes approximately constant between 0.8 and $1.8 \mathrm{kV}$, indicating efficient charge collection without amplification. Also shown in fig. 5 is the charge collection in pure argon gas. Note that the yield is reduced by about a factor of 3 owing to back-scattering of electrons from argon, and also that amplification sets in for $\left|\mathrm{V}_{\mathrm{PC}}\right|>1.5 \mathrm{kV}$.

The quantum efficiency of four solid TMAE photocathodes [calculated from Eq. (10)] is shown in fig. 6, with the TMAE gas-phase yield also shown for comparison. The curves for solid TMAE are labelled by $T_{P C}(p)$. The data were taken under the following conditions: $260(0), 260(0.045), 243$ $(0.045)$ and $220(0.045)$. Note that the yield increases with decreasing temperature. However, it was observed that when the TMAE gas flow was stopped, the higher yield curve, $260(0.045)$, decayed rapidly to the lower curve, $\mathbf{2 6 0}(0)$, indicating that the increase in efficiency is probably due to a strong gradient of TMAE gas at the cathode surface. This is still confirmed when melting a solidTMAE photocathode, as we observed in that case an increase of the current $I_{A d}$ by a factor of 2 to 3 , before it falls rapidly to the quoted limit. The yields reported in this and subsequent sections are the intrinsic photocathode efficiencies, with the small gas-phase photoionization contribution removed by eq. (10).

These cathodes may be useful for fast RICH detectors (even though the best efficiency is well below the gas-phase efficiency), because the photocathode produces an isochronous (hence fast) signal and because the photon feedback is strongly suppressed owing to the low TMAE partial 
pressure. Better cathodes for fast RICH detectors have, however, been produced, as will be shown in subsequent sections.

\subsection{Liquid-TMAE photocathodes}

A liquid-TMAE photocathode was obtained by warming a deposited solid TMAE layer to $273 \leq \mathrm{T}_{\mathrm{PC}} \leq 278 \mathrm{~K}$; however, at this temperature the photocathode evaporated rapidly. To obtain a stable response it was necessary to flow, through the test chamber, methane that contained 0.06 Torr of TMAE, i.e. nearly its equilibrium pressure. The data are shown in fig. 7 by the curves labelled 273 (0.06) and 278 (0.06). The efficiencies are similar to those for solid TMAE, and are significantly smaller than the TMAE gas-phase efficiency, which is also shown in fig. 7 for comparison.

\subsection{Adsorbed TMAE films}

In this case the bare metallic PC was kept at room temperature (298 K), and a small partial pressure ( 0.045 Torr) of TMAE-laden methane gas was flowed through the TC. The thickness of the adsorbed TMAE film could not be measured (it was adsorbed and not cryopumped); however, it was so thin that the yield depended on the substrate metal. The measured photocathode yields are shown in fig. 8a on a logarithmic scale and in fig. $8 \mathrm{~b}$ on a linear scale. The four different metal substrates-ss, copper, brass, and zinc, labelled 298 (0.045)-are shown in both fig. 8a and fig. $8 \mathrm{~b}$, whereas the bare-metal yields-298 (0)-are shown only in fig. 8a. The TMAE gas-phase efficiency is also included for comparison. An efficiency of $\sim 15 \%$ (at $185 \mathrm{~nm}$ ) obtained with the ss and zinc PCs should be compared with a yield of $\sim 10^{-4}$ for the bare metals. This enormous enhancement of more than a factor of $10^{3}$ may occur, as has been observed in refs. [12] and [1], because the TMAE adsorbed on the metal surface forms an electric dipole layer, which facilitates the electron extraction [13, 14]. The existence of a high-yield adsorbed film had previously been hypothesized in order to obtain quantitative agreement with the observed photon feedback in the DELPHI barrel RICH prototype detector [15].

All yields increased by about $10 \%$ when the TMAE partial pressure was increased by $67 \%$ to 0.075 Torr.

The adsorbed TMAE film efficiencies are high; the films are relatively easy to obtain, because the PC is at room temperature with only a small TMAE partial pressure in the gas flow. The obvious advantages are fast response, suppressed photon feedback, and room-temperature operation.

\subsection{Caesium iodide photocathode}

Reflective CsI photocathodes with high quantum efficiency have been known since 1957, through the work of Taft and Philipp [16], and have been further developed by Carruthers [17], for space astronomy. His reflective photocathodes operate by extracting electrons into vacuum. In the present work, atmospheric pressure gas extraction is required. 
To fabricate the CsI photocathode, a ss plate was mounted in a bell-jar pumped to a pressure $\mathrm{p} \leq 10^{-6}$ Torr, and $\mathrm{a} \sim 100 \mathrm{~nm}$ layer of aluminium was vacuum-evaporated onto it, followed by a layer of CsI [Merck \#2861; impurities of $\mathrm{Rb}, \mathrm{Na}, \mathrm{K}, \mathrm{Ba}, \mathrm{Li}, \mathrm{Mg}$, Sr, and $\mathrm{Ca}$ (in ppm) are 1000, 500, 500, 50, $40,10,5$, and 1 , respectively, with $0.13 \mathrm{ppm}$ of other metals]. The aluminium layer was deposited in order to insulate the CsI layer from impurities adsorbed on the ss plate. After CsI deposition, the bell-jar was filled with dry nitrogen at atmospheric pressure, and the ss plate was removed. It was rapidly wrapped in aluminium foil (to avoid exposure to sunlight) and stored in a silica-gel-filled glass dessicator, which was then pumped to a pressure $\mathrm{p} \leq 10^{-3}$ Torr, sealed, and transferred to the photon monochromator laboratory. When the time came for measurement, the dessicator was unsealed, and the CsI photocathode plate was clipped manually onto the PC plane in the test chamber. As soon as the TC was closed, it was then flushed with a fast flow of clean methane gas. The total integrated air-exposure time of the CsI PC was between 10 and $20 \mathrm{~min}$.

The $160 \mathrm{~nm}$ light transmission of the TC gas is monitored by the current $I_{T}$ with the PC in the up position. Full photon transmission is obtained in about one hour of normal gas flow $(20 \mathrm{l} / \mathrm{h})$, but it requires about 48 hours in order to obtain the full anode signal $I_{A d}$. The TC and entry lines have a volume of about 31 ; hence, with the normal gas flow, about seven volume changes occur per hour. This is consistent with the observed time needed to obtain full photon transmission (i.e. to reduce the oxygen level); however, full photocathode yield requires extended rinsing of the CsI surface.

A check of the linearity of $I_{A d}$ with $I_{T}$, made when the $160 \mathrm{~nm}$ light intensity was reduced by mechanically decreasing the height of the monochromator exit slit, is shown in fig. 9.

Five CsI photocathodes of different thicknesses $(15,30,120,250$, and $500 \mathrm{~nm})$ were deposited and measured. The yields are shown in fig. 10, with the TMAE gas-phase efficiency included for comparison. The quantum yields [calculated from eq. (8) because CsI has negligible equilibrium vapour pressure] are large, and they are close to the TMAE gas-phase efficiency, especially at the shorter wavelengths. Note that the efficiency generally increases with increasing thickness. An assumed absorption cross-section $\sigma=10 \mathrm{Mb}$ corresponds to an absorption mean free path $\mathrm{l}_{\mathrm{ph}}=100 \mathrm{~nm}$, which is probably too small since the yield is still rising even for the $500 \mathrm{~nm}$ thick PC (i.e. $51_{\mathrm{ph}}$ ). Even so, for the quantum yield to continue to increase implies that there must be efficient electron transport from deep layers to the surface. Thicker layers have not yet been made, but will be tried out in the near future.

A second $500 \mathrm{~nm}$ thick CsI photocathode has been made and compared with the first one, as shown in fig. 11. The data show the evolution of the quantum yield, measured 1, 17, and $41 \mathrm{~h}$ after the $(30 \mathrm{l} / \mathrm{h})$ gas flow started. This second cathode is perhaps the better one, but overall they are in reasonable agreement $(\sim 10 \%)$. They both give a somewhat larger yield than Carruthers' $1000 \mathrm{~nm}$ thick vacuum photocathode [17], which is also shown in fig. 11. His photocathode was deposited, transferred, installed, and pumped without rinsing with methane gas. It therefore appears either that electron extraction in methane is more efficient than vacuum extraction (in contradiction with the Appendix of ref. [12]), or that the CsI must be rinsed to obtain its maximum 
yield. The yield of this cathode remained unchanged after 3 weeks' storage under reduced pure methane gas flow $(1 / / h)$.

The best CsI photocathode yield is almost as good as that of gas-phase TMAE for $\lambda<185 \mathrm{~nm}$. It therefore represents a serious alternative to TMAE for RICH detectors, as well as for readout of the fast component of $\mathrm{BaF}_{2}$ scintillation.

Recently, Dangendorf et al. [18], in an independent measurement, have used a $1500 \mathrm{~nm}$ thick CsI reflective photocathode to detect primary and secondary scintillation photons from xenon in an $X$-ray imaging gas-scintillation counter. Their measured quantum efficiency is $9 \%$ at $170 \mathrm{~nm}$, compared with $\sim 36 \%$ in this work. Their calibration was by photon counting, with the quantum efficiency of the photomultiplier (Hamamatsu, R1460) as the reference, whereas we measure the photocurrent and use the gas-phase photoionization of TMAE $[7,8]$ as the reference. Their photocathode was deposited directly onto a copper substrate, and only pumped to $10^{-1}$ Torr for outgassing. The quality of their CsI was identical to ours since they came from the same source. We therefore believe that the above difference is caused by their photocathode not being sufficiently rinsed. As can be seen from fig. 11, the $170 \mathrm{~nm}$ yield increased by a factor of 2 during the 1 to $41 \mathrm{~h}$ period after the $(30 \mathrm{l} / \mathrm{h})$ gas flow started. We could not measure the increase in yield between 0 and $1 \mathrm{~h}$ because the TC gas was not yet sufficiently transparent.

When the $500 \mathrm{~nm}$ thick CsI photocathode was cooled slowly down to $160 \mathrm{~K}$ (the temperature of liquid xenon), the quantum yield decreased by a factor of about 100 from its room-temperature starting value. It was hypothesized that this was due to the ambient-temperature TC walls being gettered by the cold CsI cathode, which poisoned its operation. However, the photocathode always recovered its full efficiency when it returned to room temperature. To verify this supposition, the cold trap (figs. 1a,b) was cooled to about $110 \mathrm{~K}$ before the CsI cathode was cooled to $160 \mathrm{~K}$. The quantum yield then decreased by only a factor of 1.2 , in accordance with the hypothesis. The small residual gettering effect that remained will probably disappear with further cooling of the trap. On this basis, it appears that a CsI photocathode will operate with full efficiency at liquid-xenon temperatures.

\subsection{Caesium iodide + adsorbed TMAE-film photocathodes}

Because of the enormous effect that an adsorbed TMAE film has on metals (see subsection 4.4 and fig. 8a), it was decided to investigate the effect of a solid, a liquid, or an adsorbed TMAE film on a CsI photocathode. The results, with the (first) $500 \mathrm{~nm}$ thick CsI photocathode as the substrate, are shown in fig. 12. The solid, liquid, and adsorbed films were formed by flowing methane, mixed with 0.04 Torr TMAE, through the TC, with the PC plate at temperatures of 248, 274, and $303 \mathrm{~K}$, respectively. In all cases the yield was several times higher than the corresponding case where a metal substrate was used. In the case of the solid and liquid layers, the yield was lower than for the pure CsI photocathode. However, for the adsorbed layer it was higher- even more than the TMAE gas-phase yield (both of these curves are included in fig. 12 for comparison). Even more remarkable 
is the fact that the adsorbed-layer yield stayed unchanged when the TMAE partial pressure was reduced to zero, as shown by the curve labelled $303(0)$, and it remained stable even after the CsI photocathode had been flushed for three weeks with pure methane. This photocathode was later exposed to air by opening the TC for one hour. Its quantum efficiency returned to the previous level after several hours of vigorous methane gas-flow $(80 \mathrm{l} / \mathrm{h})$.

The CsI photocathode with an adsorbed TMAE film has several advantages as a RICH detector, e.g. it has a high yield, it is fast, and it is solar blind. The biggest problem in a detector with gas amplification will probably be due to photon feedback. Gas-phase feedback cannot occur since the TMAE partial pressure in the gas is negligible. Photon feedback from the photocathode iself will tend to merge with the primary avalanche if the amplifying structure (wire) is near the cathode (i.e. $0.5 \mathrm{~mm}=10 \mathrm{~ns}$ ). The amplifying gas must, of course, be opaque to the $\mathrm{C}^{*}$ (atomically excited carbon) lines at 156 and $166 \mathrm{~nm}$ (i.e. 7.95 and $7.47 \mathrm{eV}$ ). The use of pentane or hexane, with methane as the amplifying gas, assures the requisite opacity and simultaneously defines the upper energy limit of the detector response (i.e. $\sim 7 \mathrm{eV})$. The $\mathrm{C}^{*}$ emission line at $193 \mathrm{~nm}(6.42 \mathrm{eV})$ is then the only remaining source of feedback instability. It will have the same level of photon feedback as that from a triethylamine (TEA)-based photoionizing gas detector, which is sensitive only to the $156 \mathrm{~nm}$ line. The quantum efficiencies of TEA at $156 \mathrm{~nm}$ and CsI+TMAE at $193 \mathrm{~nm}$ are similar, and since these emission lines are equally probable it is expected that these two types of detectors will show a similar performance. A TEA pad-detector (with amplifying wires $0.5 \mathrm{~mm}$ from the pad plane) has recently been tested successfully [19]. It showed considerably enhanced stability to photon feedback, compared with the results obtained with a TMAE gas detector.

A pad-detector, in which the CSI+TMAE photocathode is deposited on the pad plane, is now being fabricated in order to test this cathode for applications as a RICH detector.

Tests for reading out a $\mathrm{BaF}_{2}$ scintillating-crystal calorimeter with a CsI photocathode have been made; they will be briefly described in section 5 and more completely in ref. [20].

\subsection{Ethyl ferrocene photocathode}

Quantum-yield measurements of an ethyl ferrocene (EF) photocathode in the region $\lambda>$ $190 \mathrm{~nm}$ have been published in a previous work by some of the present authors [21, 22]. This material is photosensitive and, unlike TMAE, does not react with air. The latter property may be of interest for some applications. Such a cathode has already been used as the photosensitive element in a small $\mathrm{BaF}_{2}$ calorimeter [23]. In the present work a new measurement of this cathode has been made over a wider wavelength range, as shown in fig. 13. The three curves are: i) for the bare ss PC plate at room temperature, labelled $298(0)$; ii) for a condensed photocathode at $273 \mathrm{~K}$ with the EF equilibrium partial pressure of 0.01 Torr, labelled $273(0.01)$, and iii) for the same cathode after the flow of EF has been stopped, i.e. 273 (0). In cases (ii) and (iii) the yield at the $\mathrm{BaF}_{2}$ scintillation wavelength $(\lambda \sim 193 \mathrm{~nm})$ is about $2 \%$, which is in agreement with previous work [22]. However, the EF yield is considerably smaller than any of the others tested here. 


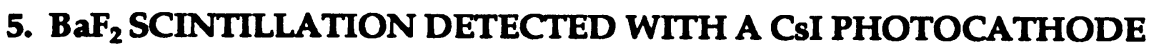

In order to independently confirm the large quantum yield of the CsI photocathode (see subsection 4.5), a home-made pulsed $100 \mathrm{kV}$ electron accelerator was used to excite fast scintillation in a $\mathrm{BaF}_{2}$ crystal, as shown in figs. 14a,b. The electron source for the accelerator starts with a pulsed (15 ns FWHM) photon beam from a low-pressure $\mathrm{H}_{2} \mathrm{UV}$ lamp with $\mathrm{CaF}_{2}$ windows. The pulsed light source is focused by a $\mathrm{CaF}_{2}$ lens through a $\mathrm{CaF}_{2}$ window and onto a $\mathrm{CsI}$ reflective vacuum photocathode placed at the beginning of the $40 \mathrm{~cm}$ long, $100 \mathrm{kV}$, accelerating structure, shown in fig. 14a. The number of photons per pulse could be regulated by an adjustable iris located in front of the UV lamp (not shown in fig. 14a). The maximum electron intensity is $\sim 10^{7}$ electrons per pulse having a maximum kinetic energy of $100 \mathrm{keV}$ corresponding to a maximum total excitation energy of $1 \mathrm{TeV}$ per pulse. The minimum measurable excitation energy is $\sim 1 \mathrm{MeV}$. The number of electrons in the beam is monitored by a copper Faraday-plate collimator, which intercepts about $75 \%$ of the beam and transmits the remainder to the $\mathrm{BaF}_{2}$ crystal. In a separate study, with a second Faraday plate (placed to intercept the collimated electron beam), it was shown that the charge measured on the Faraday-plate collimator determines the transmitted charge with a fractional r.m.s. error of about $0.3 \%$. The electron kinetic energy is determined by the power supply voltage corrected for energy loss in a $12 \mu \mathrm{m}$ Mylar entrance window. A more complete description of this device will be given in ref. [20].

The $\mathrm{BaF}_{2}$ crystal was mounted to intercept the collimated electron beam, and the (second) $500 \mathrm{~nm}$ thick CsI photocathode was positioned to view the scintillation source, as shown in fig. 14b. The refraction-corrected solid angle subtended by the photocathode was $\Delta \Omega / 4 \pi=2.44 \%$. A fast signal was observed on the collection grid of a low-noise charge-sensitive preamplifier $(\langle\mathrm{NEC}\rangle \approx$ 300 electrons; $400 \mathrm{mV} / \mathrm{pC}$ ) followed by a shaper (ELSCINT model CAU-N3; $0.8 \mu$ s shaping time) (see fig. 15a). The signal (normalized to a constant charge input by the Faraday-plate monitor) varied linearly with the electron kinetic energy as shown in fig. 15b. The slope of this curve, the solid angle, the crystal transmission (0.8), and the charge input $\left(2.65 \times 10^{5}\right.$ electrons per pulse) determine a total $(4 \pi)$ signal of 40 photoelectrons per $\mathrm{MeV}$ of deposited energy.

In a separate study of $\mathrm{BaF}_{2}$ scintillation, a total emission efficiency of 560 photons per $\mathrm{MeV}$ for the fast component and 9600 photons per MeV for the slow component was measured using two different photomultiplier detectors (Hamamatsu R1460 and 1332Q). The observed pulse waveform is shown in fig. 16, where the fast and slow signals may be clearly discerned. The lifetime of the slow component was measured to be $740 \mathrm{~ns}$, whereas the pulse reflects only the $15 \mathrm{~ns}$ excitation pulse width.

The measured quantum-yield spectrum of this CsI photocathode (fig. 11) has been averaged over the fast $\mathrm{BaF}_{2}$ scintillation spectrum [24] to give an average yield $\langle\mathrm{Q}\rangle=5.75 \%$. This yield, together with the measured fast emission yield of 560 photons per $\mathrm{MeV}$, then predicts a cotal $(4 \pi)$ signal of 32 photoelectrons per $\mathrm{MeV}$, assuming that no reflectivity occurs onto the other faces of the 
crystal, hence in rough agreement with the measured value of 40 and also confirming the large CsI yield.

Note that with a reflective cathode the maximum observable signal is limited, by total internal reflection, to about $11.6 \%$ per face, i.e. to 4.6 photoelectrons per $\mathrm{MeV}$ per face. A six-faced detector (or a one-faced detector with each of the other five faces mirrored) could then obtain a total of 28 photoelectrons per $\mathrm{MeV}$ with perfect crystal transmission. A way to overcome the loss of photons, which is due to internal reflection, could be the insertion, between the crystal and the CsI photocathode, of a liquid interface such as TMS or TMP, which would transmit the fast $\mathrm{BaF}_{2}$ scintillation component. Such a test is under preparation.

A transmissive photocathode, deposited directly on the crystal's surface, would avoid the problem of losses due to total reflection, but it would not increase the detected signal because its quantum yield is only $10 \%$ of the reflective cathode yield [17]. It would also require a transmissive conducting film, deposited directly on the $\mathrm{BaF}_{2}$ crystal, as a substrate for the transmissive CsI photocathode. Such a conducting film would engender additional photon absorption.

The use of the reflective CsI+TMAE adsorbed film cathode will increase the quantum yield by a factor of 2 (at $193 \mathrm{~nm}$ ), to give a quite respectable detected signal of 9.2 photoelectrons per MeV per face, and hence a total of 56 photoelectrons per $\mathrm{MeV}$.

In comparison, the TMAE gas-phase quantum yield [7, 8] has been averaged over the fast $\mathrm{BaF}_{2}$ scintillation spectrum [24] to give $\langle\mathrm{Q}\rangle=9.84 \%$, hence a total $(4 \pi)$ signal of 55 and a detectable signal of 6.4 photoelectrons per MeV per face. Therefore, the response from the CsI+TMAE adsorbed film cathode should be $44 \%$ larger than that from gas-phase TMAE.

Other ways of increasing the signal require that the scintillation spectrum be shifted to about $170 \mathrm{~nm}$ [25] where the CsI+TMAE quantum yield is high $(\sim 46 \%)$. The peak of the liquid-xenon emission spectrum is, in fact, at $170 \mathrm{~nm}$, and its scintillation efficiency is enormous, i.e. about (2-3) $\times$ $10^{4}$ photons per $\mathrm{MeV}$, corresponding to a potential total signal of (10-15) photoelectrons per keV. This potential signal could be fully realized because the photocathode would be in direct contact with the liquid, which means that total reflection losses could be avoided. However, the photoelectrons must be efficiently injected into the liquid, where they would drift (in an applied electric field) to a collection grid for detection.

\section{SUMMARY AND CONCLUSIONS}

An investigation of reflective photocathodes has shown that electron emission into methane gas is as efficient as emission into vacuum, and that the use of TMAE adsorbed films can increase the electron yields significantly. These cathodes must be rinsed by gas flow in order to obtain optimal yield. A specific CsI+TMAE adsorbed-film photocathode has been developed, which has $46 \%$ quantum efficiency at $\mathbf{1 7 0} \mathrm{nm}$ and is well adapted for fast RICH detectors because it has isochronous signals, reduced photon feedback, and room-temperature operation. This cathode also promises to 
allow the simple, efficient, and cheap readout of fast inorganic UV scintillators such as $\mathrm{BaF}_{2}$, as well as of the noble liquid scintillators.

\section{Acknowledgements}

The work reported here is part of the LAA project; it was also supported by the Institut National de Physique Nucléaire et de Physique des Particules (IN2P3) of France. Y. Giomataris wishes to acknowledge the support of the National Research Centre Demokritos (NRCD), Athens. We wish to thank G. Passardi for assisting in the construction of the apparatus, and M. Bosteels for his help with the gas system. Thanks are due also to A. Brehm and C. Nichols for their excellent vacuum evaporations, to J.-P. Jobez for the design of the apparatus, and to R. Saigne and J.L. Escourrou for its assembly and modifications. J.C. Santiard provided us with the low-noise preamplifier, and gave us the benefit of his expertise. 
[1] V. Peskov, G. Charpak, P. Miné, F. Sauli, D. Scigocki, J. Séguinot, W.F. Schmidt and T. Ypsilantis, Nucl. Instrum. Methods A269 (1988) 149.

[2] J. Séguinot, Fast RICH detectors for LHC/SSC/ELOISATRON, Proc. Symp. on Particle Identification at High-Luminosity Hadron Colliders, Batavia, Ill., 1989, eds. T.J. Gourlay and J.G. Morfin (Fermilab, Batavia, 1989), pp. 215-253 and 671-683.

T. Ypsilantis, Particle identification at hadron colliders, ibid., pp. 133-158 and/or preprint CERN-EP/89-150 (1989).

J. Séguinot and T. Ypsilantis, report CERN-LAA/89-1, pp. 327-340.

[3] G. Charpak, V. Peskov and D. Scigocki, ibid., pp. 139-167.

[4] J. Séguinot and T. Ypsilantis, ibid., pp. 187-199.

[5] T. Ypsilantis, Proc. ECFA Study Week on Instrumentation Technology, Barcelona, 1989, eds. E. Fernandez and G. Jarlskog (CERN 89-10, Geneva, 1989), Vol. 2, pp. 661-674.

[6] P. Baillon, Y. Declais, M. Ferro-Luzzi, B. French, P. Jenni, J.-M. Perreau, J. Séguinot and T. Ypsilantis, Nucl. Instrum. Methods 126 (1975) 13.

[7] R.A. Holroyd, J.M. Preses, C.L. Woody and R.A. Johnson, Nucl. Instrum. Methods A261 (1987) 440.

[8] R. Arnold, P. Baillon, H.J. Besch, M. Bosteels, E. Christophel, M. Dracos, Y. Giomataris, J.L. Guyonnet, G. Passardi, P. Petroff, J. Séguinot and T. Ypsilantis, Nucl. Instrum. Methods A270 (1988) 289.

[9] W. Kluge, in Landolt-Börnstein (Springer, Berlin, 1959), Vol. 2, Part 6.

R. Schulze, Z. Phys. 92 (1934) 223.

[10] D.F. Anderson, Nucl. Instrum. Methods 126 (1975) 13 and 178 (1980) 125; IEEE. Trans. Nucl. Sci. NS-28 (1981) 842.

[11] Y. Giomataris, CERN-DELPHI 86-17, RICH15 (1986).

[12] D.F. Anderson, R. Bouclier, G. Charpak and S. Majewski, with Appendix by G. Kellner, Nucl. Instrum. Methods 217 (1983) 217.

[13] D.P. Woodruff and T.A. Delchar, Modern techniques of surface science (University Press, Cambridge, 1986).

[14] D.A. King and D.P. Woodruff in: The chemical physics of solid surface and heterogeneous catalysis (Elsevier, Amsterdam, 1984), Vol 3, Part B.

[15] R. Arnold, P. Baillon, H.J. Besch, M. Bosteels, E. Christophel, M. Dracos, Y. Giomataris, J.L. Guyonnet, G. Passardi, P. Petroff, J. Séguinot, D. Toët, J. Tocqueville and T. Ypsilantis, Nucl. Instrum. Methods A270 (1988) 255.

[16] E.A. Taft and H.R. Philipp, Phys. Chem. Solids 3 (1957) 1.

[17] G.R. Carruthers, Appl. Opt. 8 (1969) 633, 12 (1973) 2501, and 14 (1975) 1667. 
[18] V. Dangendorf, A. Breskin, R. Chechik and H. Schmidt-Bocking, Weizmann Inst. preprint WIS-89/81/December/PH, presented at SPIE 1990, Symp. on Instrumentation in Astronomy, Tucson (Ariz.), 1990. Also Nucl. Instrum. Methods A289 (1990) 322.

[19] R. Arnold, Y. Giomataris, J.L. Guyonnet, J. Séguinot and T. Ypsilantis, report in preparation.

[20] J. Séguinot, M. Bosteels, Y. Giomataris, G. Passardi, V. Peskov, J. Tischhauser and T. Ypsilantis, report in preparation.

[21] G. Charpak, V. Peskov, F. Sauli and D. Scigocki, Nucl. Instrum. Methods A270 (1988) 255.

[22] G. Charpak, D. Lamb, V. Peskov, D. Scigocki and J. Valbis, see Proc. of Ref. [5], Vol. 2, pp. 593-598.

[23] V. Peskov, G. Charpak, F. Sauli, D. Scigocki, V. Diep and D. Janjic, Nucl. Instrum. Methods A283 (1989) 786.

[24] P. Schotanus et al., IEEE Trans. Nucl. Sci. NS-34 (1987) 272.

[25] P. Schotanus et al., IEEE Trans. Nucl. Sci. NS-36 (1989) 132.

Also A.F. Buzulutskov et al., Nucl. Instrum. Methods A288 (1990) 659. 
Figure captions

Fig. 1a: The experimental layout of the photon monochromator (PMC) with the $D_{2}$ UV lamp (La), the $\mathrm{CaF}_{2}$ lens (Le), the $\mathrm{CaF}_{2}$ beam splitter (BS), the reflected beam photomultiplier (PMR), and the $\mathrm{CaF}_{2}$ window (W). The test chamber (TC) is shown in more detail in fig. $1 b$.

Fig. 1b: The test chamber contains the mesh cathode (MC), the wire anode (A), the photocathode (PC) with its cooling loop, the cold trap, and the transmitted beam photomultiplier (PMT). The glass window of PMT (and PMR) is coated with a $1 \mu \mathrm{m}$ thick vacuum-deposited film of the wavelength shifter (WLS) p-terphenyl. Not shown in the figure are the gas-flow inlet and outlet.

Fig. 2a: The measured product of quantum efficiency and gain $Q_{T} G_{T}$ of the transmitted beam photomultiplier (PMT) divided by the rated gain $g=1.4 \times 10^{5}$ at $1.4 \mathrm{kV}$ versus the wavelength $\lambda$.

Fig. 2b: The anode-collected photocathode current $\mathrm{I}_{\mathrm{Au}}$ in a methane-TMAE (0.04 Torr) mixture as a function of the photocathode voltage $\mathrm{V}_{\mathrm{PC}}$, for two different PMT voltages, at $\lambda=$ $160 \mathrm{~nm}$ (0 suppressed).

Fig. 3: The measured quantum yield $Q$ of four bare metals versus the photon wavelength $\lambda$.

Fig. 4: The transmission TR of TMAE-laden methane gas in the TC for two temperatures of the $P C$ plate versus gas flow-time $t$. In the case of $T_{P C}=260 \mathrm{~K}$ the TMAE gas injection started at $\mathrm{t}=0$ and stopped at $\mathrm{t}=20 \mathrm{~min}$, whereas at $\mathrm{T}_{\mathrm{PC}}=298 \mathrm{~K}$ the TMAE gas injection was not stopped.

Fig. 5: The anode-collected photocathode current $I_{A}$ versus the photocathode voltage $V_{P C}$ for electron emission into methane or argon.

Fig. 6: The measured quantum yield $Q$ versus the photon wavelength $\lambda$ for four solid-TMAE photocathodes. The labelling in the top right-hand corner gives the photocathode temperature $(\mathrm{K})$, followed by the TMAE partial pressure (Torr) in brackets. The solid line without data points is the TMAE gas-phase quantum efficiency. 
Fig. 7: The measured quantum yield $Q$ versus the photon wavelength $\lambda$ for two liquid-TMAE photocathodes. As in fig. 6, the labelling gives the photocathode temperature (K), followed by the TMAE partial pressure (Torr) in brackets. The solid line without data points is the TMAE gas-phase quantum efficiency.

Fig. 8a: The measured quantum yield $Q$ versus the photon wavelength $\lambda$ for four metal cathodes with an adsorbed TMAE film. The labelling gives the photocathode temperature (K), followed by the TMAE partial pressure (Torr) in brackets. The solid line without data points is the TMAE gas-phase quantum efficiency. The open points are from fig. 3.

Fig. 8b: The same TMAE adsorbed film data as in fig. 8a, but on a linear scale.

Fig. 9: The linearity of the anode-collected photocathode current $I_{A}$ versus the transmitted beam monitor current $\mathrm{I}_{\mathrm{T}}$ (with the PC plate in the up position). The PMC light intensity was varied by adjusting the height of the monochromator exit slit.

Fig. 10: The measured quantum yield $Q$ versus the photon wavelength $\lambda$ for five CsI photocathodes of different thicknesses. The solid line is the TMAE gas-phase quantum efficiency.

Fig. 11: The measured quantum yields $Q$ versus the photon wavelength $\lambda$ of the second $500 \mathrm{~nm}$ thick CsI photocathode after 1, 17, and 41 hours of methane gas-flow through the TC. The solid line is the TMAE gas-phase yield, the dashed line is the first $500 \mathrm{~nm}$ thick CsI photocathode yield, and the dash-dotted line is the Carruthers vacuum photocathode yield [17].

Fig. 12: The measured quantum yield $Q$ versus the photon wavelength $\lambda$ of the first $500 \mathrm{~nm}$ thick CsI photocathode, onto which a solid, a liquid, or an adsorbed film of TMAE has been deposited. The labelling gives the photocathode temperature (K), followed by the TMAE partial pressure (Torr) in brackets. The solid line is the TMAE gas-phase quantum efficiency and the dashed line is the CsI yield.

Fig. 13: The measured quantum yields $Q$ versus the photon wavelength $\lambda$ of the stainless-steel cathode with an adsorbed ethyl ferrocene (EF) film. The labelling gives the photocathode temperature (K), followed by the TMAE partial pressure (Torr) in brackets. The solid line is the TMAE gas-phase quantum efficiency. 
Fig. 14a: A schematic view of the $100 \mathrm{kV}$ electron accelerator with the pulsed $\mathrm{H}_{2}$ light source, the CsI photocathode, the accelerating structure, and the copper Faraday-plate collimator.

Fig. 14b: A schematic view of the $\mathrm{BaF}_{2}$ scintillator, the CsI reflective photocathode, the collection mesh, the charge-sensitive preamplifier, and the $0.8 \mu \mathrm{s}$ shaper.

Fig. 15a: Typical CsI photocathode signal observed, at the output of the shaper, with a digital oscilloscope Tektronix 2440, for $5 \mathrm{GeV}$ total energy deposited in the $\mathrm{BaF}_{2}$ crystal. The horizontal and the vertical scales are $1 \mu$ s and $2 \mathrm{mV}$ per division, respectively.

Fig. 15b: The CsI photocathode-detected signal versus the incident electron kinetic energy $\mathrm{K}_{\mathrm{e}}$ The signal is normalized to a constant charge input of $2.65 \times 10^{5}$ electrons per pulse, hence the $100 \mathrm{keV}$ electron kinetic energy point corresponds to $26.5 \mathrm{GeV}$ of total energy deposited in the $\mathrm{BaF}_{2}$ crystal.

Fig. 16: The $\mathrm{BaF}_{2}$ scintillation pulse waveform observed with a Hamamatsu R1460 photomultiplier with a $\mathrm{Cs}_{2} \mathrm{Te}$ solar-blind photocathode. The photomultiplier pulse amplitude ( $1 \mathrm{mV}$ per division) is shown versus time ( $50 \mathrm{~ns}$ per division). The fast pulse width reflects only the excitation pulse width ( $15 \mathrm{~ns}$ FWHM), whereas the slow pulse has a decay time of $740 \mathrm{~ns}$. The $\mathrm{Cs}_{2} \mathrm{Te}$ photocathode is more efficient for the fast pulse $(193 \mathrm{~nm})$, hence the relative amplitudes of the fast and slow components are biased. 


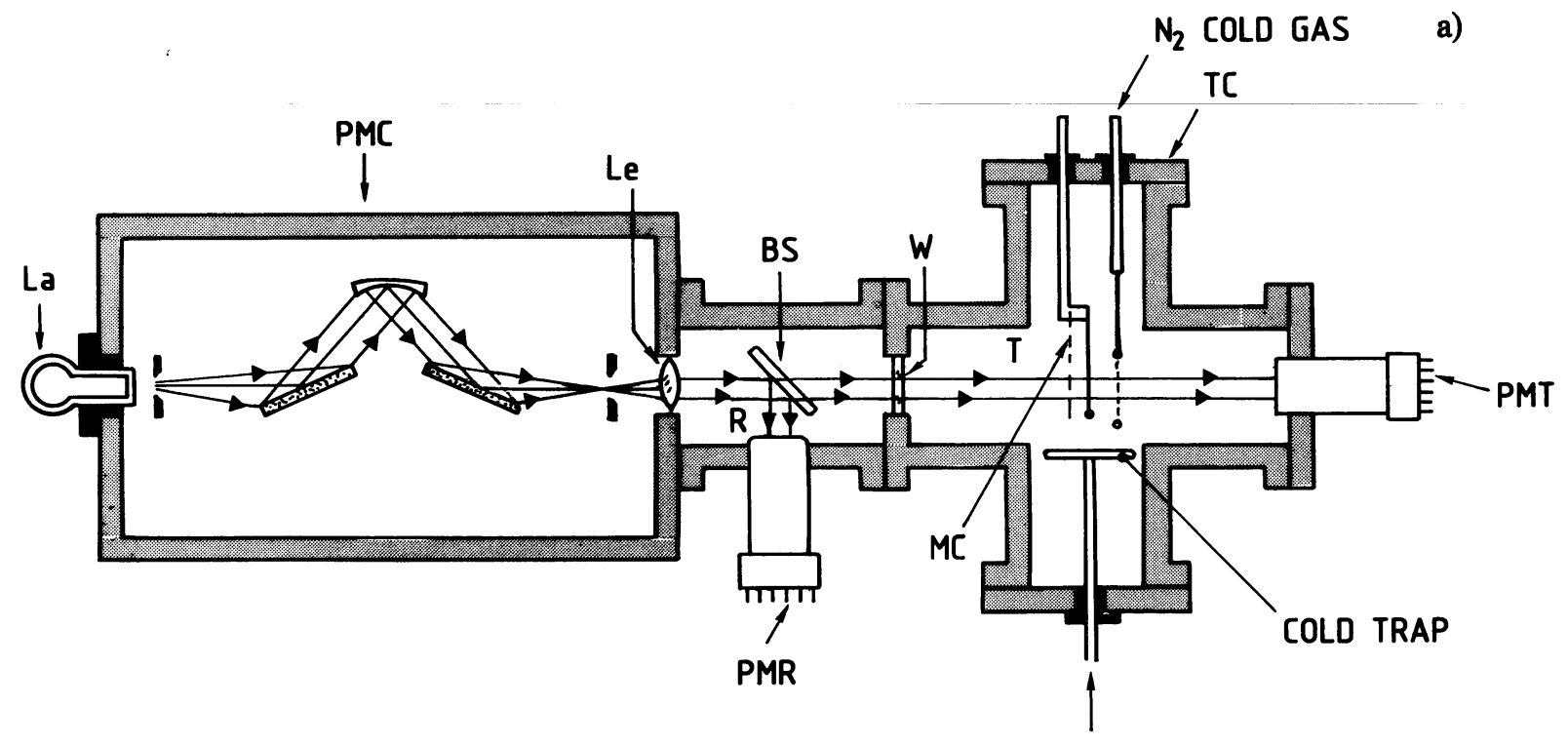

$\mathrm{N}_{2}$ COLD GAS

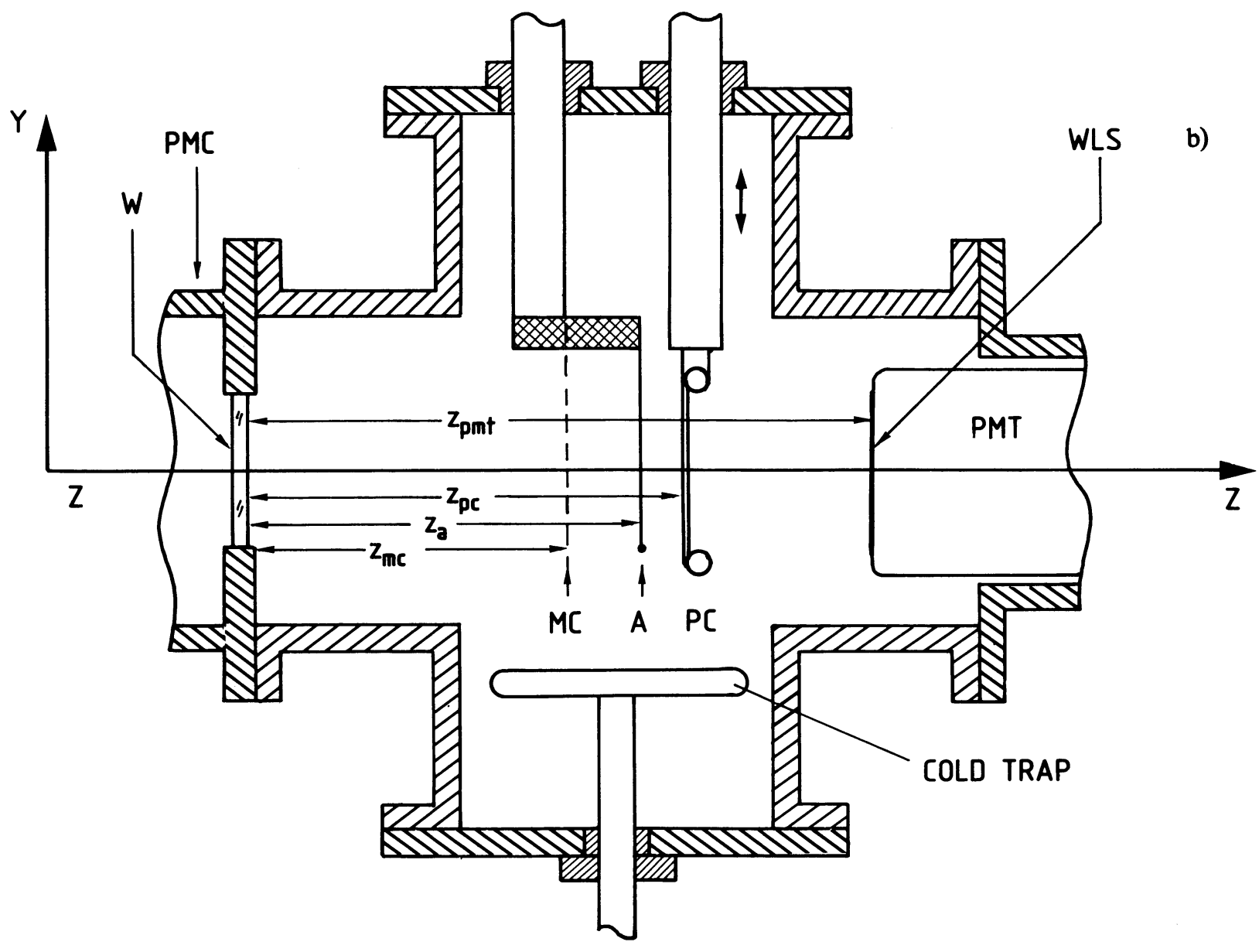

Fig. 1 

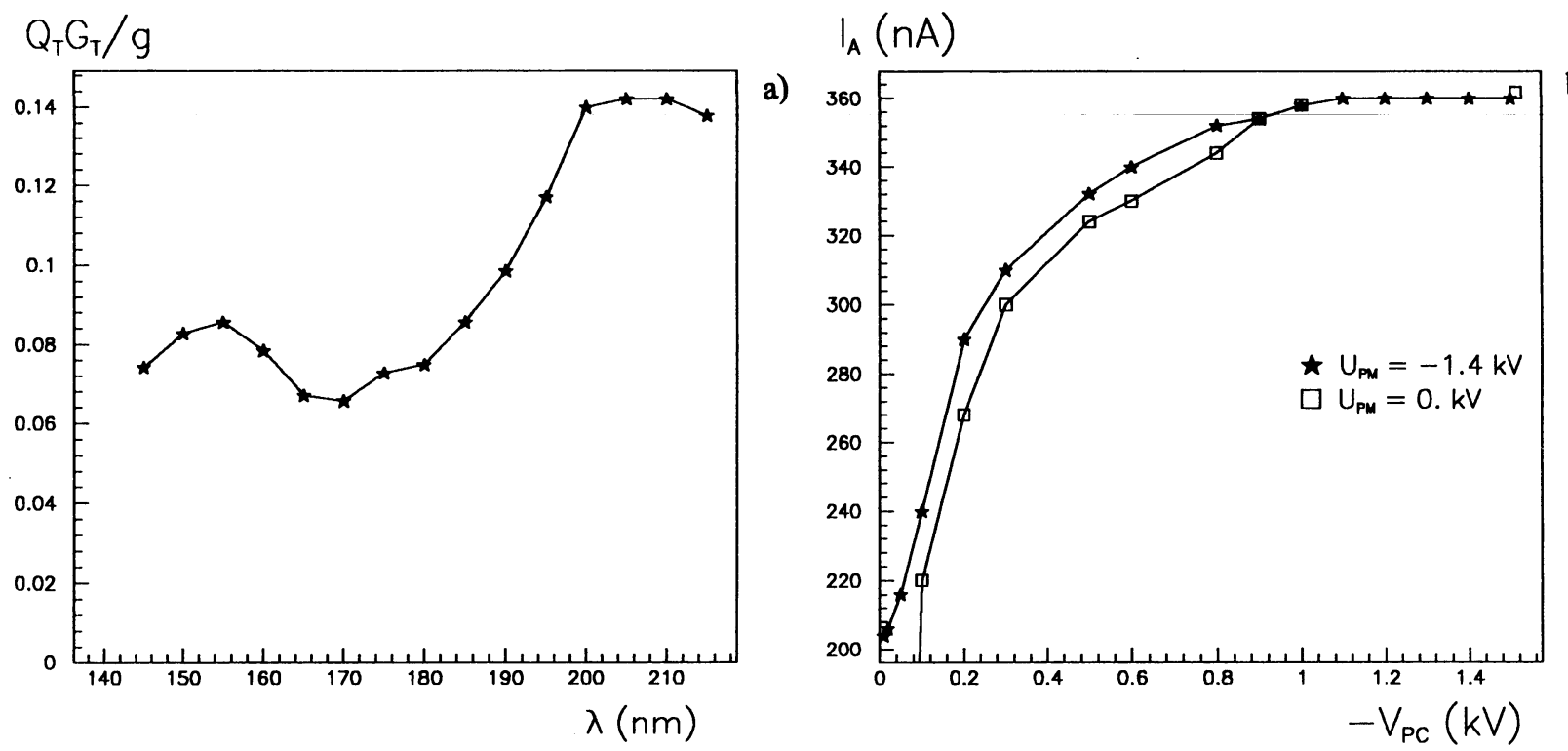

b)

Fig. 2

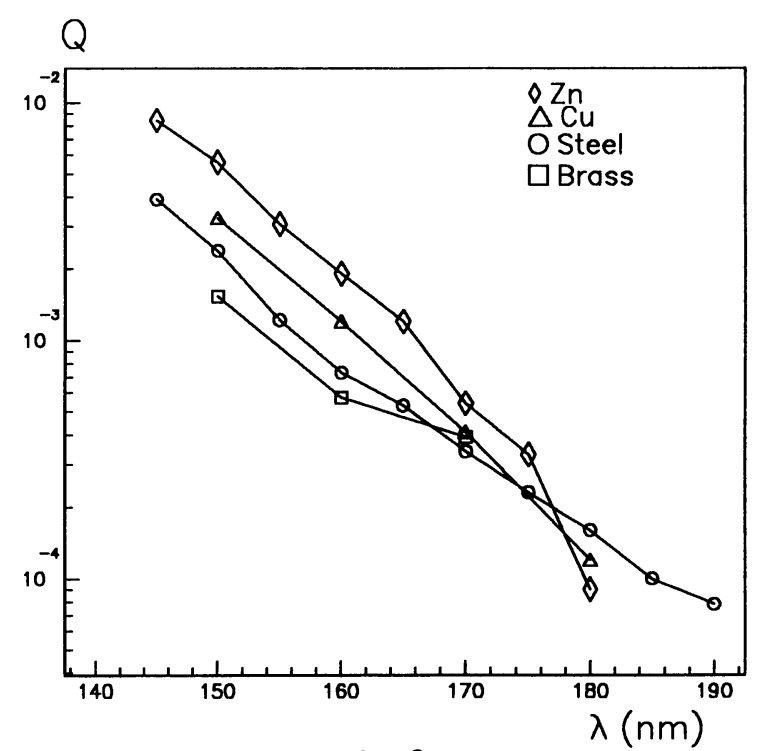

Fig. 3

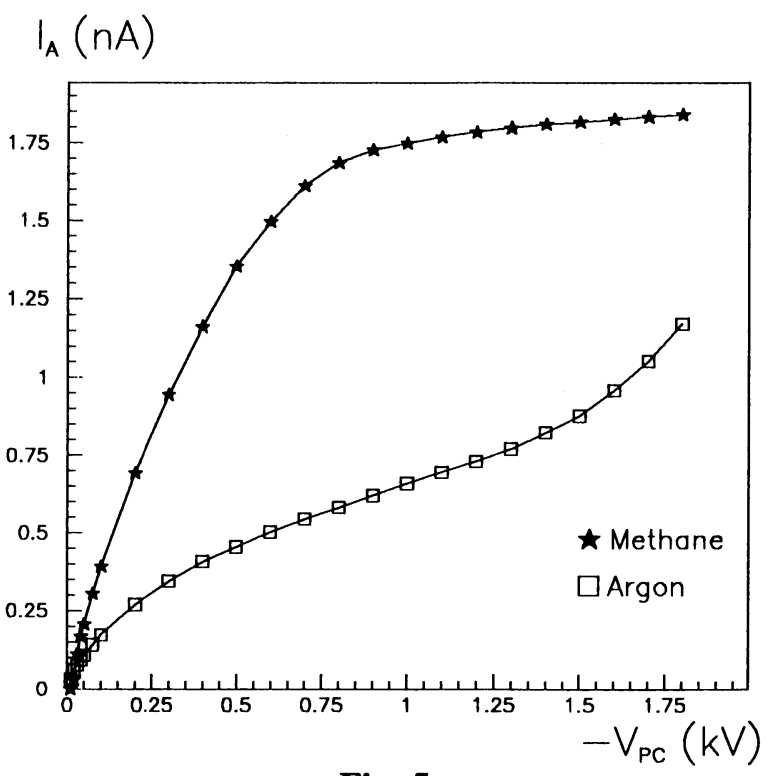

Fig. 5

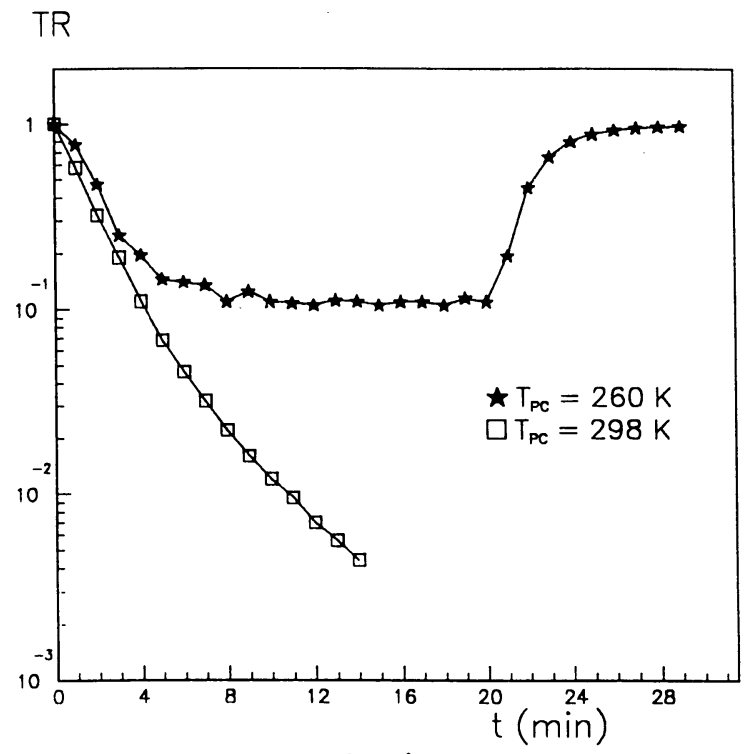

Fig. 4

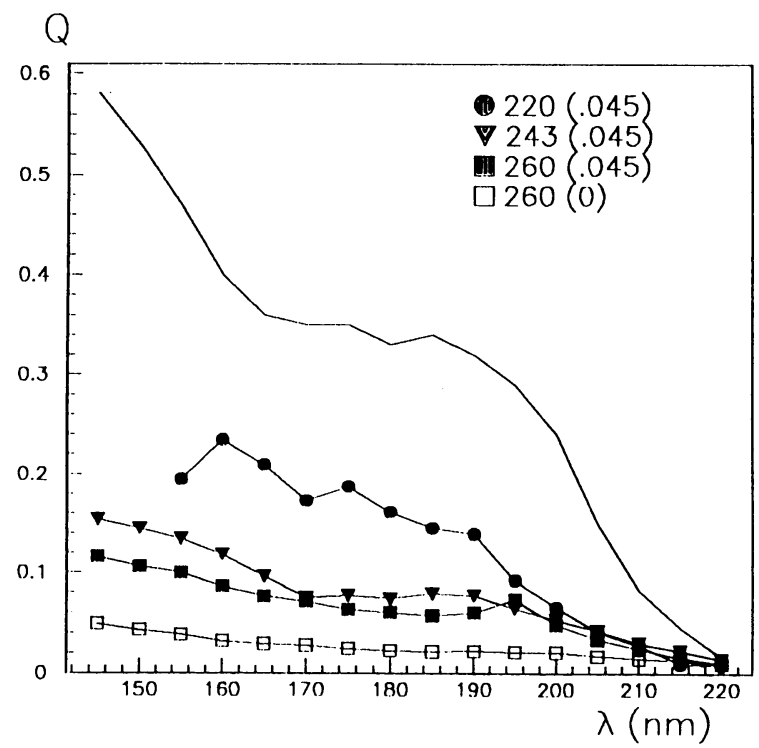

Fig. 6 


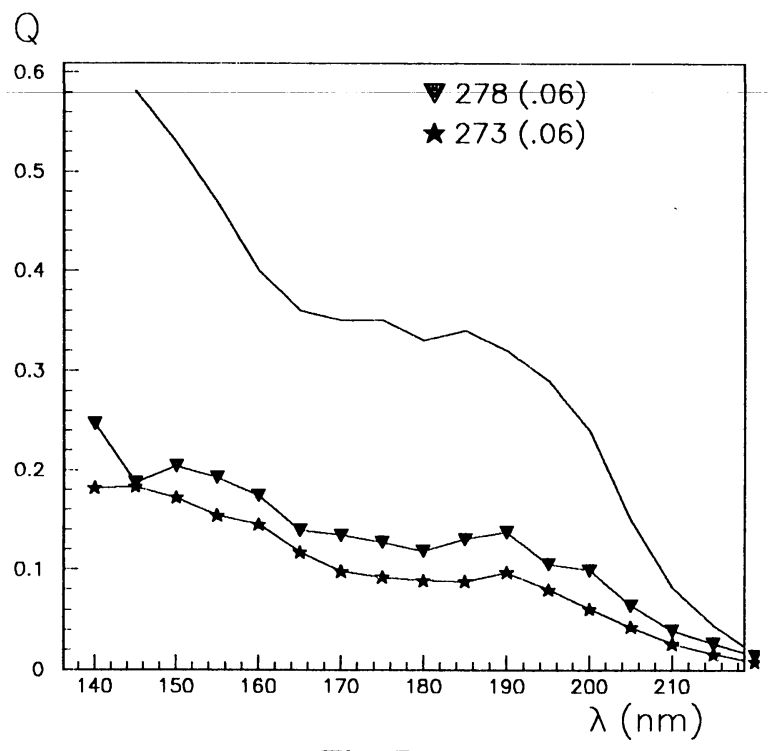

Fig. 7

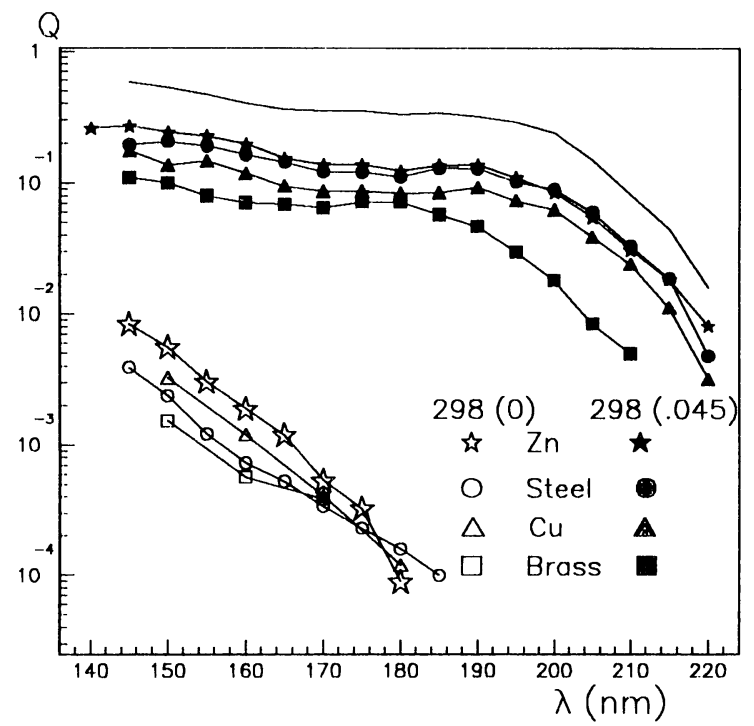

a)

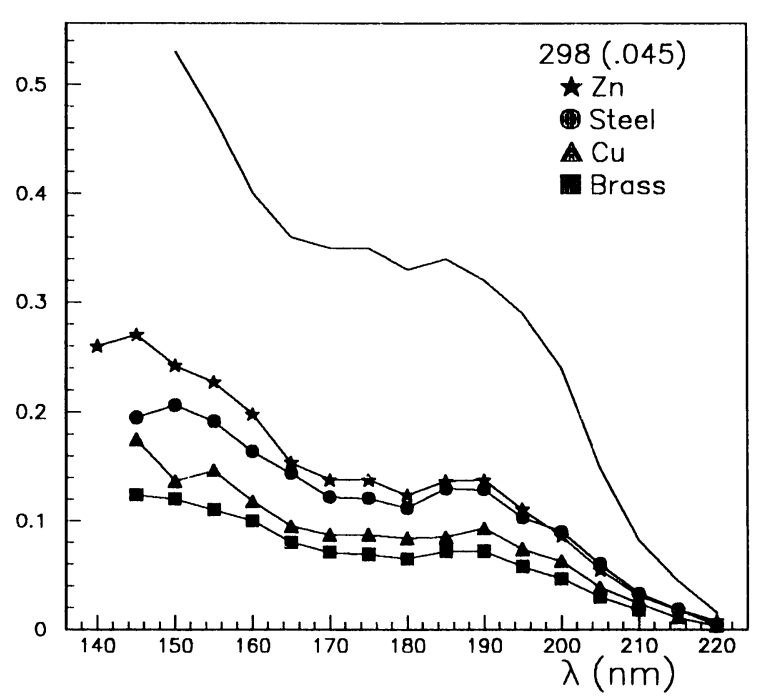

b)

Fig. 8

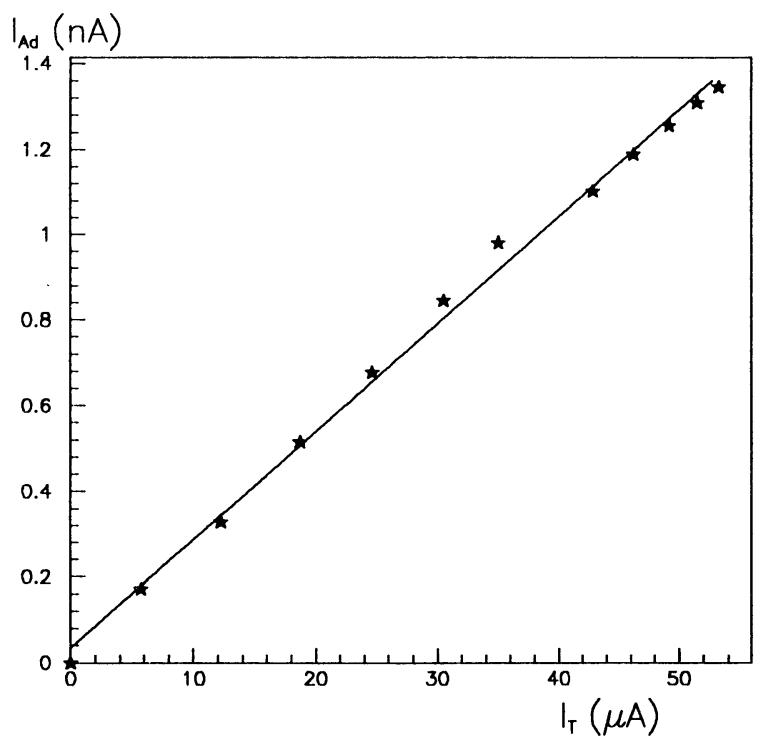

Fig. 9

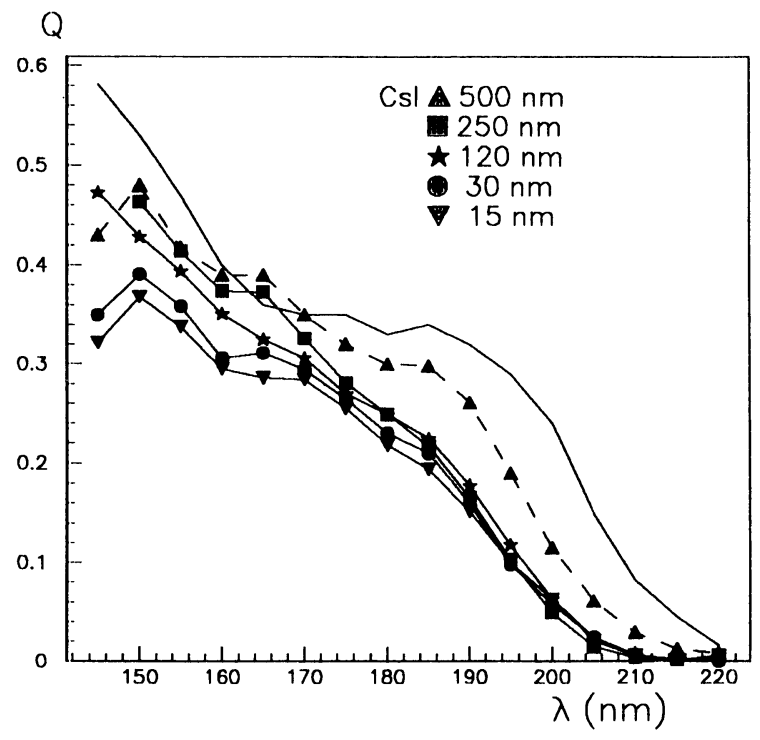

Fig. 10 


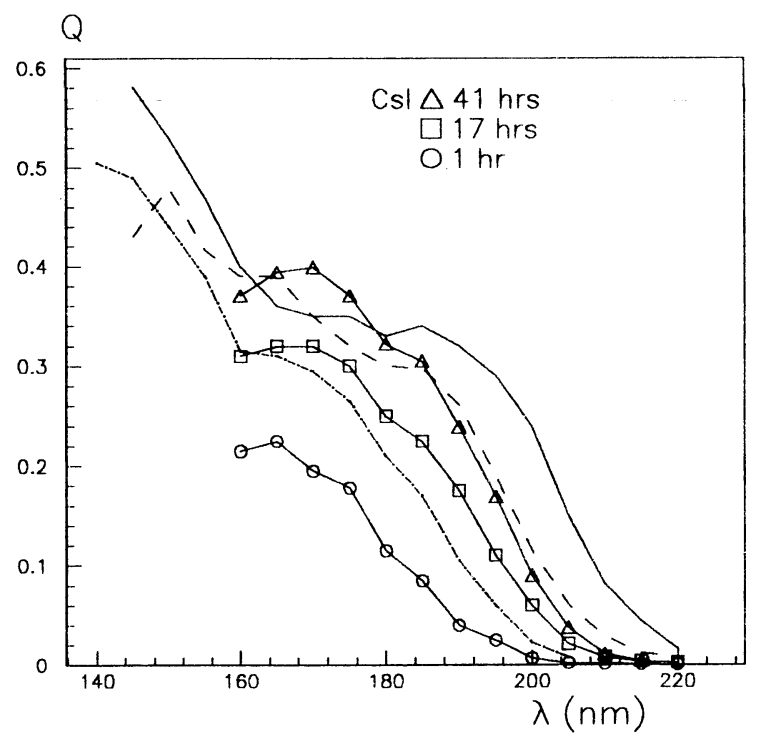

Fig. 11
Q

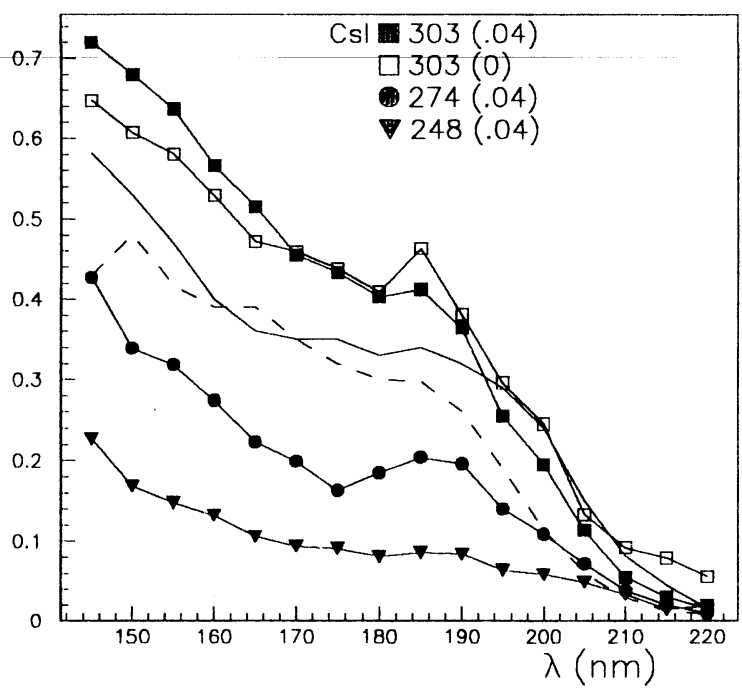

Fig. 12

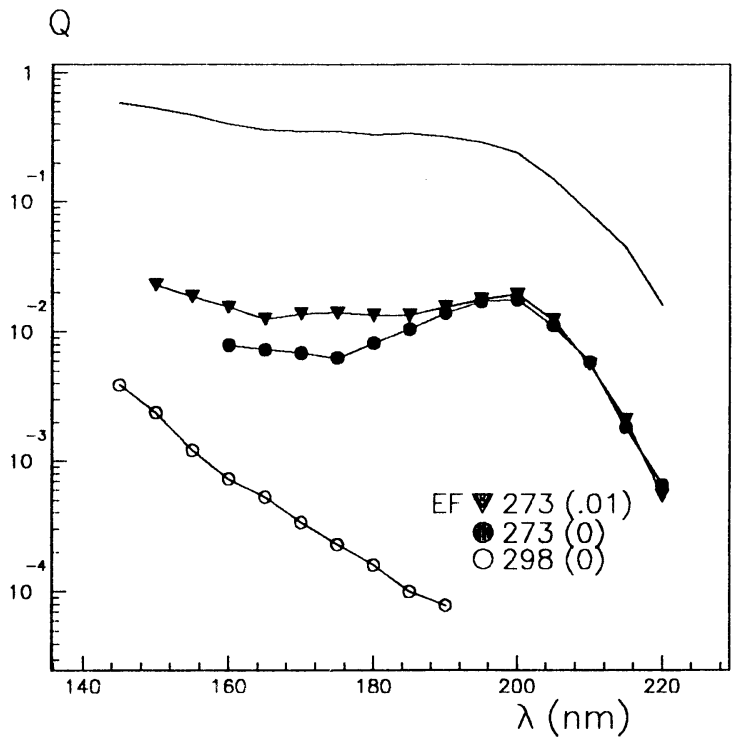

Fig. 13 

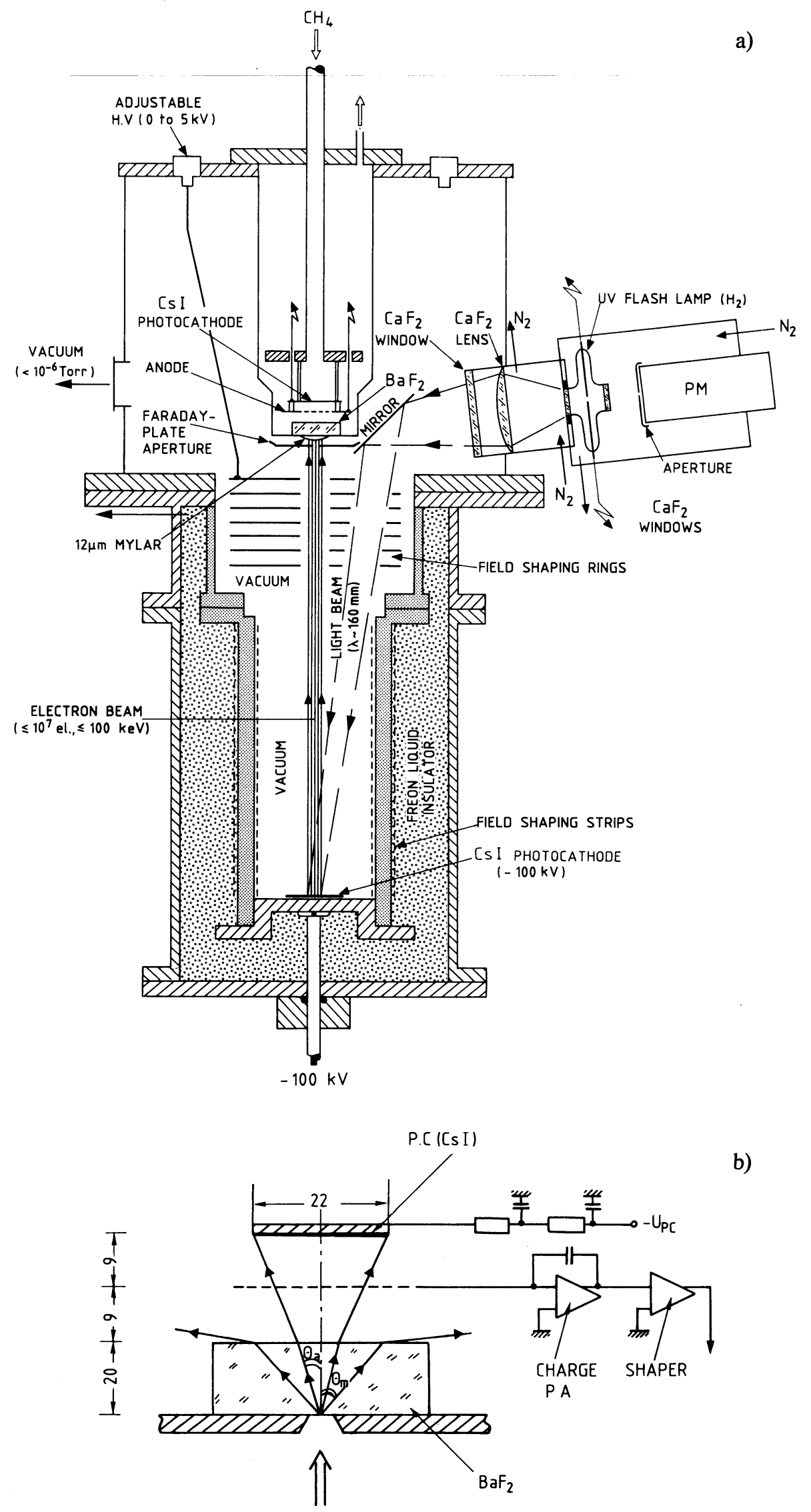

ELECTRON BEAM

Fig. 14 


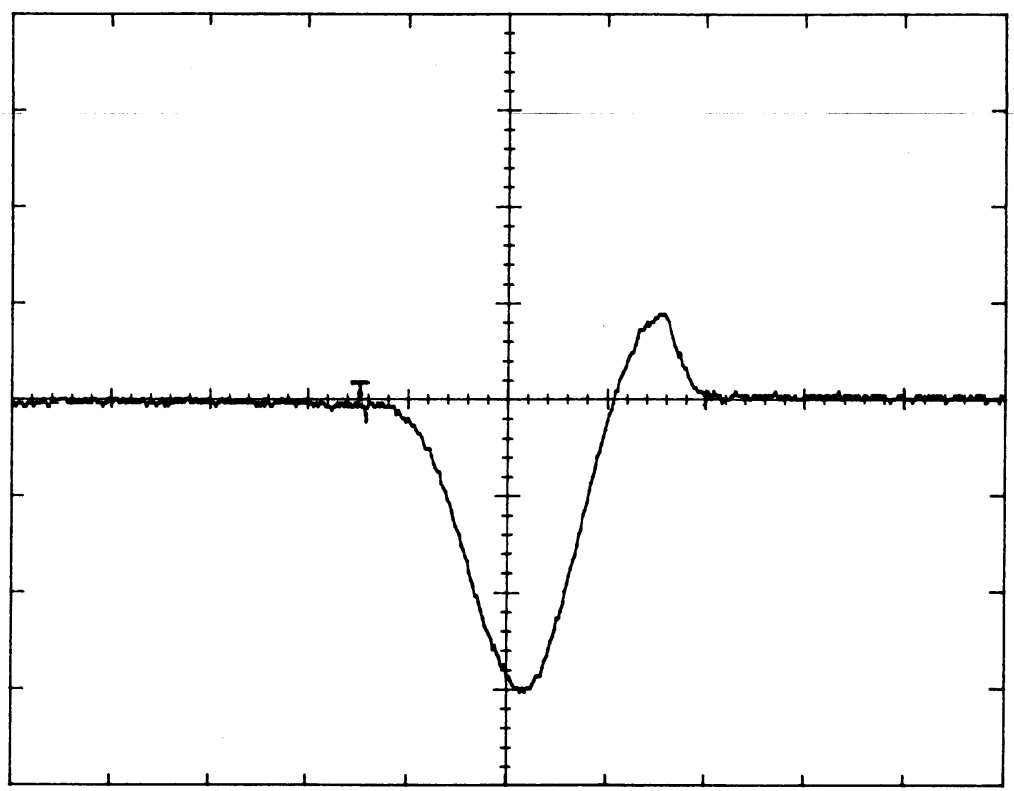

a)

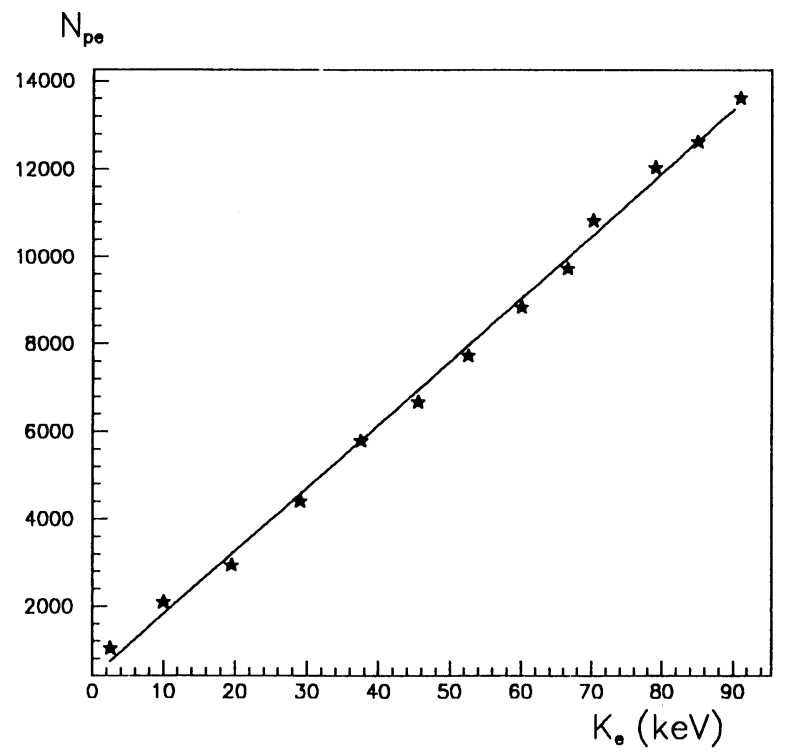

b)

Fig. 15

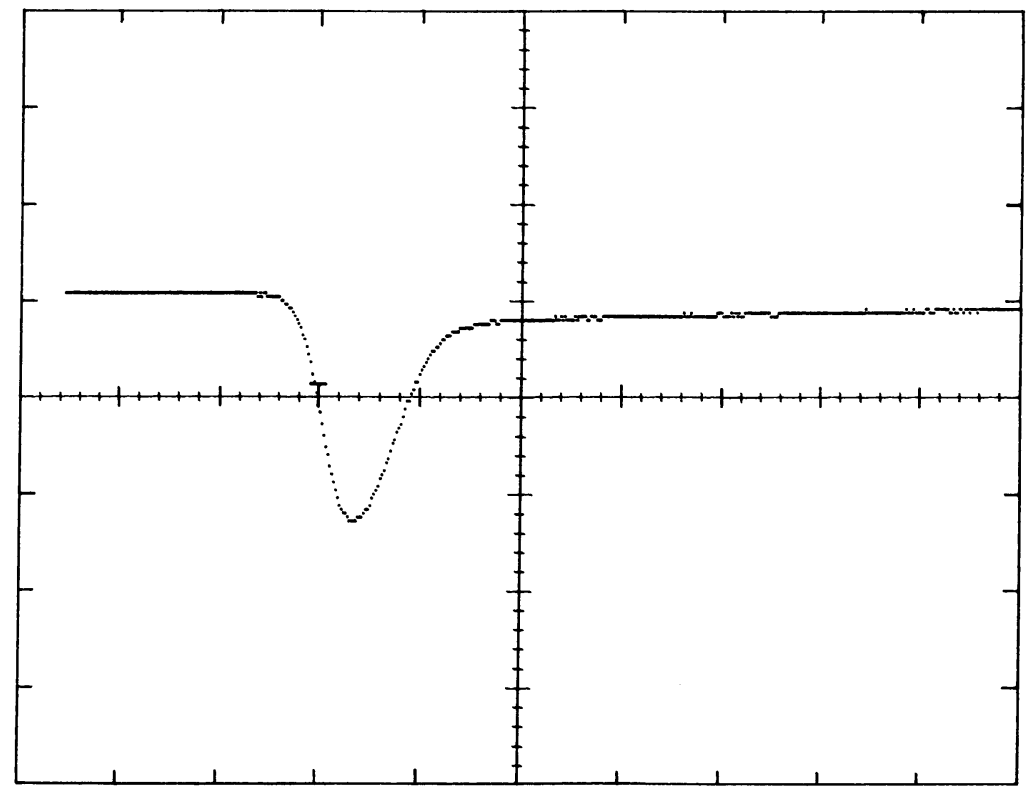

Fig. 16

Imprime au CERN. Prix : 0.85 Fr. 